Article

\title{
Statins Improve the Long-term Survival in the Survived Sepsis Patients, a Nationwide Cohort Study in Taiwan
}

\section{Statin and long-term prognosis of sepsis}

\author{
Ming-Shun Hsieh ${ }^{1-4}$, Shu-Hui Liao ${ }^{5}$, Vivian Chia-Rong Hsieh ${ }^{6}$, Chorng-Kuang How ${ }^{3,4}$, Jen-Huai \\ Chiang ${ }^{6}$, Sung-Yuan Hu ${ }^{7}$, Yi-Tzu Lee ${ }^{3,4}$, Chen-June Seak ${ }^{8}$ and Pau-Chung Chen ${ }^{1,9, *}$ \\ 1 Institute of Occupational Medicine and Industrial Hygiene, National Taiwan University, College of Public \\ Health, Taipei, Taiwan; edmingshun@gmail.com \\ 2 Department of Emergency Medicine, Taipei Veterans General Hospital, Taoyuan Branch, Taoyuan, \\ Taiwan; \\ 3 Department of Emergency Medicine, Taipei Veterans General Hospital, Taipei, Taiwan; \\ ckhow@vghtpe.gov.tw \\ 4 School of Medicine, National Yang-Ming University, Taipei, Taiwan; yitzulee@gmail.com \\ 5 Department of Pathology and Laboratory, Taipei Veterans General Hospital, Taoyuan Branch, Taoyuan, \\ Taiwan; selina620626@yahoo.com.tw \\ 6 Department of Health Services Administration, China Medical University, Taichung, Taiwan; \\ hsiehchiarong@gmail.com (V.C.-R.H.); zinvii@gmail.com (J.H.C.) \\ 7 Department of Emergency Medicine, Taichung Veterans General Hospital, Taichung, Taiwan; \\ song9168@pie.com.tw \\ 8 Department of Emergency Medicine, Lin-Kou Medical Center, Chang Gung Memorial Hospital, Taoyuan, \\ Taiwan; julianseak@hotmail.com \\ 9 Department of Environmental and Occupational Medicine, National Taiwan University College of \\ Medicine and Hospital, Taipei, Taiwan; \\ * Correspondence: pchen@ntu.edu.tw
}

\begin{abstract}
:
Background:

Most sepsis patients died during their first episode and the long-term survival post discharge was low. Major adverse cardiovascular events and repeated infections were regarded as the major causes in such conditions. No definite medications were proven to effectively improve the long-term outcome. We aimed to examine the benefit of statins on the long-term outcome of survived sepsis patients.

Methods:

Between 1999 and 2013, a total of 220,082 patients with the first episode of sepsis hospitalization were included and 134,448 ones (61.09\%) survived to discharge. The survived patients who subsequently had statins use of $>30$ cumulative defined daily doses (cDDDs) post discharge were defined as the statin users. After propensity score matching of 1:5, a total of 7,356 and 36,780 survived patients were retrieved as the study (statin-users) and comparison cohort (non-statin users), respectively. The main outcome was the long term survival post discharge. HR with $95 \% \mathrm{CI}$ was calculated using the Cox regression model to evaluate the effectiveness of statins with further stratification analyses according to cDDDs.
\end{abstract}

Results: 
The study cohort, that is, the statin users had an adjusted HR of 0.29 (95\% CI, 0.27-0.31) in long term mortality rate compared with the comparison cohort. For statin users with the cDDDs of 30-180, $180-365$, and $>365$, the adjusted HRs were $0.32,0.22$, and 0.16 , respectively, (95\% CI, $0.30-0.34,0.19-$ 0.26 , and $0.12-0.23$, respectively) compared with the non-statin users (defined as statins use $<30$ cDDDs post discharge), and the $\mathrm{P}$ for trend $<.0001$. In the sensitivity analysis, after excluding the survived patients who died within three and six months post discharge, the adjusted HR of statins use remained significant $(0.35,95 \%$ CI $0.32-0.37$ and $0.42,95 \%$ CI $0.39-0.45$, respectively).

Conclusions:

Statins decreased the long-term mortality post sepsis. Further randomized control trial deserved to be conducted to confirm this observation.

Keywords: 3-hydroxy-3-methylglutaryl-coenzyme-A inhibitors; Sepsis

\section{Introduction}

Sepsis, a complex syndrome caused by infections with an unregulated immune response, is a leading cause of mortality worldwide.[1-3] Despite the advance in medical care, the mortality rate remains high and ranged from $17 \%-26 \%$ in varied severity and countries.[2] It has been demonstrated that the survived sepsis patients had an increased subsequent long-term risk of major cardiovascular events (MACEs) and associated worse survival rate compared with the general population.[4-6] Maintenance of long-term post-sepsis survival and being free from its complications, such as MACEs or recurrent infections remains a great challenge.[7-9] Currently, few studies proposed effective interventions or medications to prevent this compromised course.

Statins (3-hydroxy-3-methylglutaryl-coenzyme-A inhibitors) are widely used in treating hypercholesterolemia and therefore reduce the risk of cardio- and cerebral-vascular diseases. Statins have also been proven to have anti-inflammation and immunomodulation effects, including the reduction of inflammatory cytokines, chemotaxis and neutrophil migration.[10-11] Because of the proposed pleiotropic immunomodulatory effects of statins, a large number of observational studies and randomized control trials were conducted thereafter, from 2004 to 2013.[12] However, the effect of statins remains a major controversy; most RCTs showed no benefit of statins in mortality during sepsis, but observational studies showed protective effect. Recently, because of the new publication of bactericidal effects of statins, and decreased risk of mycobacterium tuberculosis infection, the use of statins in sepsis raised a new attention.[13] Furthermore, it was also proposed in recent days that different type of statins had exerted varied sepsis protection effects and explored a new prospective.[14]

In light of rising incidence and falling fatality rate, to improve the long-term outcomes among the sepsis survivors is increasingly important. In this current study, we hypothesized that statin use may improve the long-term outcome in the first-episode of survived sepsis patients via its potential secondary prevention in cardiovascular and cerebrovascular diseases and recurrent infections. This nationwide population-based cohort study was conducted by using the National Health Insurance Research Database of Taiwan (NHIRD).

\section{Methods}

\section{Data sources and study participants}

We conducted this nationwide cohort study using data from the NHIRD. The National Health Insurance program was launched in 1995 in Taiwan by the National Health Insurance Administration (NHIA) and it provided coverage for $>23.03$ million residents $(99.2 \%$ of the entire population). The NHIA released identification-encrypted claims data to the National Health Research Institute and 
established the NHIRD. The data confidentiality was strictly maintained in accordance with the regulations of NHIRD.

In the NHIRD, the diagnosis codes of the International Classification of Diseases, Ninth Revision, Clinical Modification (ICD-9-CM) were used to identify specific diagnoses. Sepsis patients were retrieved from the database by using the ICD-9-CM codes 038, 995.91, and A038. The specificity of the diagnosis of sepsis in the NHIRD has been validated in previous studies. ${ }^{7}$ The statins of interest in this study was retrieved from the claims data by ATC codes of C10AA, C10BA, and C10BX.

The first episode of sepsis necessitating admission was defined as the index hospitalization throughout the study. The index date was defined as the discharge date of the index hospitalization for sepsis. The associated comorbid conditions were also identified by using the ICD-9-CM codes; the diagnoses prior to or in concurrence with the index hospitalization for sepsis were considered as the underlying comorbidities.

The patients were regarded as taking a certain kind of drugs if they took them for more than one month within a three month period prior to the index hospitalization. The immunosupressants included the followings: cyclosporin, everolimus, mycophenolic acid, sirolimus, and tacrolimus were also taken into consideration in this current study.

We defined the specific managements or procedures during the hospitalization by using the claims information for insurance charge and these included (1) inotropic agent use, (2) intensive care unit (ICU) admission (claims codes of 02011K, 02012A, 02013B, 03010E, 03011F, 03012G, 03047E, 03048F, 03049G in the NHIRD), and (3) receiving mechanical ventilation (claims code of 57001B).

Initially, we used the NHIRD to retrieve the survived sepsis patients of first episode from 1999 to 2012. The claims data of NHIRD was from 1999 to 2013 and therefore all the included patients were allowed to be followed up at least for one year.

In this current study, we categorized the statin users into four groups according to the cumulative Defined Daily Doses (cDDDs): $<30$, 30-180, 180-365, and >365 cDDDs during the followup period because the duration of the refill card in Taiwan was 3 months. The study cohort was defined to have the statin use for $\geq 30$ cDDDs after surviving the first episode of sepsis and the cDDDs was calculated from the index date. The comparison cohort was composed of the non-statin users or statin users of $<30$ cDDDs. In this current study, we also defined the statin user of $<30$ cDDDs as the non-statin users.

To avoid the bias from selection, we matched the study and comparison cohort patients by using the propensity score matching. The propensity scores used in this study were composed of multiple variables of interest, such as, age, sex, socioeconomic status, urbanization level, baseline comorbidities, calculated via logistic regression. Propensity score matching could reduce the selection bias because it was capable of bundling many confounding covariates that are presented in an observational study.

Data for socioeconomic status, urbanization level, and residential area were obtained directly from the database. We used the paid insurance premiums as a proxy for household income level and the socio-economic backgrounds. We further classified these into total four categories. Those with well-defined monthly wages were grouped into three categories: (1) less than NT\$ (New Taiwan dollar) 20000, (2) NT\$ 20000 to NT\$ 40000, (3) NT\$ 40000 to NT\$ 60000 and (4) more than NT\$ 60000.

The participant selection process of the nationwide databases was showed in Supplement Figure 1. The sepsis patients aged $<20$ or $>100$ years and those infected with human immunodeficiency virus were excluded from this study. Since the NHIRD contains de-identified secondary data for research, our study was exempted from the requirement of informed consent from participants. This study was approved by the Institutional Review Board of China Medical University (CMUH104-REC2-115).

\section{Main Outcomes and Measures}

The main outcome was the long-term survival after the index hospitalization between the study (statin users of $\geq 30$ cDDDs) and the comparison cohorts (non-users or statin users of $<30 \mathrm{cDDDs}$ ).

Further comparisons were conducted to compare the long-term survival within the study cohorts with different cumulative dose (cDDDs of 30-180, 180-365, and >365). Hazard ratios (HRs) 
with $95 \%$ confidence interval (95\% CI) were calculated using the Cox proportional hazards regression model.

\section{Sensitivity analysis}

Since most sepsis patient readmitted and died soon after discharge, and we mainly focused on the long-term protective effect of statins, we further conducted the sensitivity analyses. We excluded the survived first episode sepsis patients who died within (1) 3 months and (2) 6 months.

\section{Statistical analyses}

Differences in demographic characteristics, comorbidities, medications, and socioeconomic status were examined by using $\chi 2$ tests for non-continuous variables and two-sample Student's ttests for continuous variables. Hazard ratios (HRs) with 95\% confidence intervals (CIs) were calculated for each variable using Cox proportional hazards regression. Adjusted HRs for mortality were obtained after adjustment for possible confounders. The Kaplan-Meier analyses with log-rank test were conducted to compare the long-term survival rate between the study and comparison cohorts.

The statistical analyses were performed using SAS 9.4 statistical package (SAS Institute Inc., Cary, NC, USA). A forest plot was created using Microsoft Excel 2010 (Microsoft, Redmond, WA, USA). A $P$ value of 0.05 was considered significant.

\section{Results}

From the NHIRD between 1999 and 2012, we initially retrieved 220,082 first episode sepsis patients. Only a total of 134,444 patients $(134,444$ of $220,082,61.09 \%)$ survived to discharge and was further included in the study. After propensity score matching of 1:5, we retrieved 7,356 patients as the study cohort and another 36,780 ones as the comparison cohorts.

Table 1 summarized the demographic characteristics, income, urbanization, Charlson comorbidity index score (CCI score), baseline comorbidities, and medications between the two cohorts after propensity score matching. The study cohort and comparison cohort had a mean (median) follow-up period of 3.83 (3.12) years and 3.26 (2.05) years, respectively.

Table 1 Demographic characteristics of the study and comparison cohorts composed of propensity score-matched patients who survived their first episode of sepsis.

\begin{tabular}{|c|c|c|c|}
\hline \multirow{3}{*}{ Variables } & $\begin{array}{c}\text { Nonuser of } \\
\text { statin }\end{array}$ & Statin user & \multirow{3}{*}{ Standardized mean difference } \\
\hline & $\begin{array}{c}\text { or users with } \\
<30 \text { cDDD } \\
(n=36780)\end{array}$ & $\begin{array}{l}\geq 30 \text { cDDD } \\
(n=7356)\end{array}$ & \\
\hline & $\%$ & n $\%$ & \\
\hline
\end{tabular}

Sex

Female

Male

Age group, years

$$
\begin{aligned}
& \text { 18-40 years } \\
& 40-60 \text { years } \\
& 60-80 \text { years } \\
& >80 \text { years } \\
& \text { Mean }(\mathrm{SD})
\end{aligned}
$$

0.011

0.011

$\begin{array}{lllll}17463 & 47.48 & 3534 & 48.04 & 0.011\end{array}$

$\begin{array}{ccccc}2097 & 5.7 & 201 & 2.73 & 0.148 \\ 9119 & 24.79 & 1927 & 26.2 & 0.032 \\ 16993 & 46.2 & 3987 & 54.2 & 0.16 \\ 8571 & 23.3 & 1241 & 16.87 & 0.161 \\ 67.39(15.43) & 67.27(13.05) & 0.008\end{array}$




\begin{tabular}{|c|c|c|c|c|c|}
\hline \multicolumn{6}{|c|}{ Insurance premium (NT dollars) } \\
\hline$<20000$ & 19632 & 53.38 & 3795 & 51.59 & 0.036 \\
\hline $20000-40000$ & 13793 & 37.50 & 2940 & 39.97 & 0.051 \\
\hline $40000-60000$ & 2448 & 6.66 & 480 & 6.53 & 0.005 \\
\hline$>60000$ & 907 & 2.47 & 141 & 1.92 & 0.038 \\
\hline \multicolumn{6}{|l|}{ Urbanization level } \\
\hline 1 (highest) & 9339 & 25.39 & 1964 & 26.7 & 0.03 \\
\hline 2 & 10631 & 28.9 & 2105 & 28.62 & 0.006 \\
\hline 3 & 5650 & 15.36 & 1133 & 15.4 & 0.001 \\
\hline 4 & 5786 & 15.73 & 1116 & 15.17 & 0.015 \\
\hline 5 (lowest) & 5374 & 14.61 & 1038 & 14.11 & 0.014 \\
\hline \multicolumn{6}{|l|}{ CCI score } \\
\hline 0 & 930 & 2.53 & 93 & 1.26 & 0.093 \\
\hline 1 & 2100 & 5.71 & 477 & 6.48 & 0.032 \\
\hline 2 & 2109 & 5.73 & 534 & 7.26 & 0.062 \\
\hline 3 & 2165 & 5.89 & 556 & 7.56 & 0.067 \\
\hline$\geq 4$ & 29476 & 80.14 & 5696 & 77.43 & 0.066 \\
\hline \multicolumn{6}{|l|}{ Baseline comorbidity } \\
\hline HTN & 28435 & 77.31 & 5666 & 77.03 & 0.007 \\
\hline Hyperlipidemia & 24574 & 66.81 & 4892 & 66.50 & 0.007 \\
\hline $\mathrm{CHF}$ & 9478 & 25.77 & 1812 & 24.63 & 0.026 \\
\hline COPD & 15963 & 43.40 & 3094 & 42.06 & 0.027 \\
\hline CLD & 12619 & 34.31 & 2452 & 33.33 & 0.021 \\
\hline CKD & 14087 & 38.30 & 2787 & 37.89 & 0.009 \\
\hline IHD & 17634 & 47.94 & 3444 & 46.82 & 0.023 \\
\hline Cancer & 8158 & 22.18 & 1630 & 22.16 & 0.001 \\
\hline $\mathrm{DM}$ & 27459 & 74.66 & 5461 & 74.24 & 0.01 \\
\hline \multicolumn{6}{|l|}{ Drugs } \\
\hline Aspirin & 4688 & 12.75 & 1043 & 14.18 & 0.042 \\
\hline NSAID & 26476 & 71.98 & 5473 & 74.4 & 0.055 \\
\hline Steroid & 15856 & 43.11 & 2278 & 30.97 & 0.253 \\
\hline Immunosuppressant & 138 & 0.38 & 67 & 0.91 & 0.067 \\
\hline
\end{tabular}

Abbreviations: CCI: Charlson comorbidity index; CKD: Chronic kidney disease; CLD: Chronic liver disease; COPD: Chronic obstructive pulmonary disease, HTN: Hypertension; ICU: Intensive care unit; IHD: Ischemic heart disease; PAOD: Peripheral arterial occlusion disease

During the follow-up period, the incidence rate of mortality in the study and comparison cohort was 0.1 and 0.29 per 1,000 person-days, respectively. In the univariate analysis of Cox regression model, the crude HR was 0.32 (95\%CI 0.3-0.34) for the statin use. After adjusting for the use of statins (defined as $\geq 30$ cDDDs use), age, gender, income, urbanization, and baseline comorbidities in the further multivariate analysis, the study cohort had an adjusted HR of 0.29 (0.27-0.31) in long-term mortality by referring to the comparison cohort.

Within the study cohort, the statin users with a cDDDs of 30-180, 180-365, and $>365$ during the follow-up period, the adjusted HRs for long-term mortality were 0.32 (95\% CI, $0.30-0.34), 0.22$ (95\% CI, 0.19-0.26), and 0.16 (95\% CI, 0.12-0.23), respectively, and presented in a dose-response manner ( $P$ 
for trend <.0001) (Figure 1). In the Kaplain-Meier analysis, we found a better long-term survival rate in the study cohort with a log-rank test of $P<0.0001$ (Figure $2 \mathrm{~A}$ ). In the stratification analysis according to the cumulative statin dose, the Kaplan-Meier analysis also showed an increased long-term survival rate in the higher dose user groups (log-rank test of $\mathrm{P}<0.0001$ ) (Figure 2B).

\begin{tabular}{|c|c|c|c|c|}
\hline \multirow{2}{*}{ Characteristics } & \multirow{2}{*}{$P$ for trend $<.0001$} & \multicolumn{3}{|c|}{ Adjusted model } \\
\hline & & OR & $(95 \% \mathrm{Cl})$ & P-value \\
\hline Non-Statin users & & 1.00 & Reference & \\
\hline \multicolumn{5}{|l|}{ Statin users } \\
\hline $30-180$ cDDDs & $\bullet$ & 0.32 & $(0.30-0.34)$ & $<.001$ \\
\hline $180-365$ cDDDs & $\diamond$ & 0.22 & $(0.19-0.26)$ & $<.001$ \\
\hline$>365$ cDDDs & $\diamond$ & 0.16 & $(0.12-0.23)$ & $<.001$ \\
\hline
\end{tabular}

$\begin{array}{lllllllll}\text { Adjusted OR } & 0 & 0.2 & 0.4 & 0.6 & 0.8 & 1 & 1.2 & 1.4\end{array}$

Model : adjusted for statin users and potential confounders

Figure 1 The forest plot showed the adjusted HRs of three groups of users: cumulative Defined Daily Doses (cDDDs) of 30-180, 180-365, and $>365$, compared with the comparison cohort of non-user or statin use $<30$ cDDDs. 

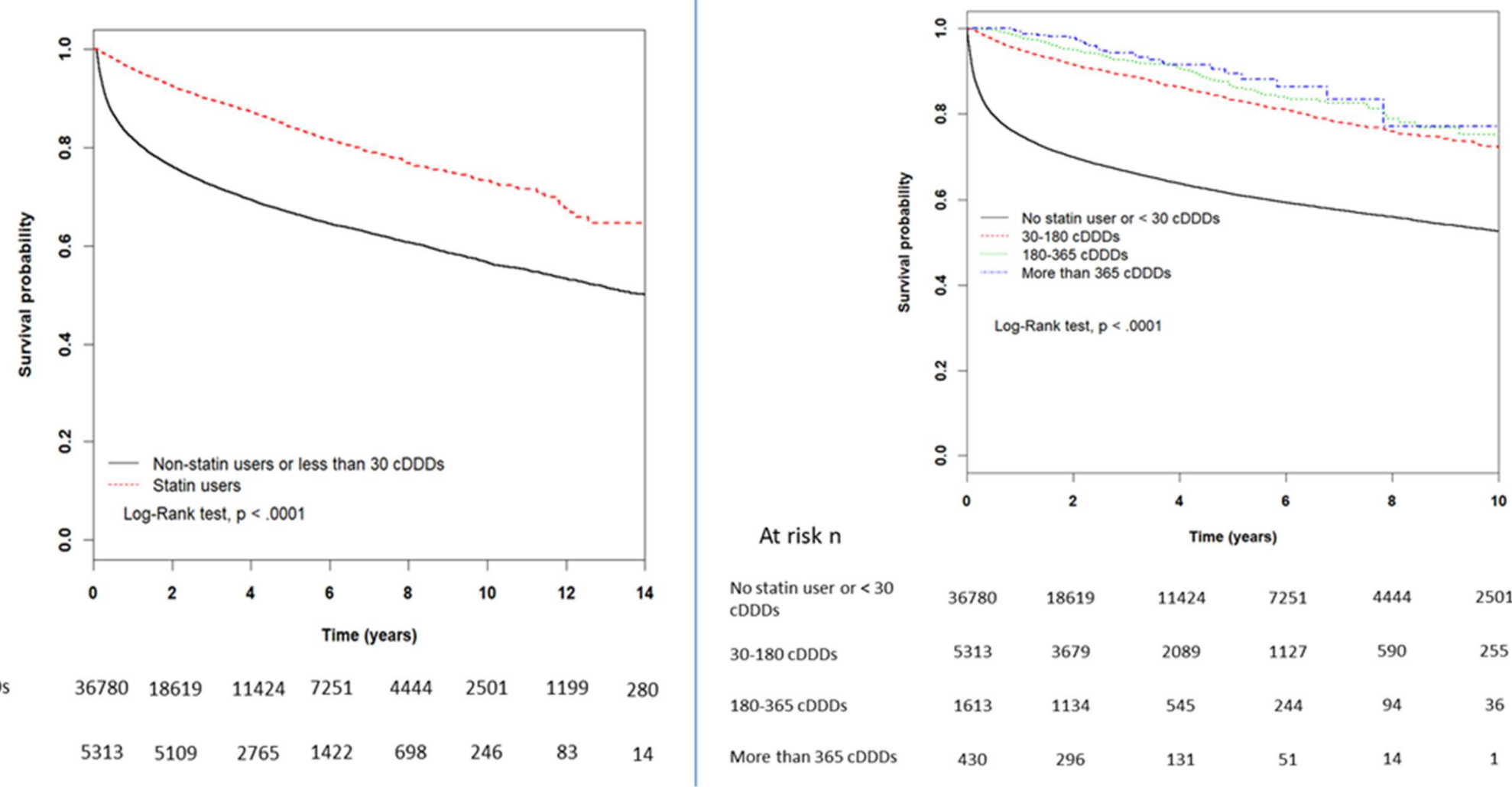

At risk $n$

No statin user or $<30$ cDDDs

30-180 CDDDs

180-365 cDDDs

More than $365 \mathrm{cDDDs}$

$\begin{array}{llllll}36780 & 18619 & 11424 & 7251 & 4444 & 2501 \\ 5313 & 3679 & 2089 & 1127 & 590 & 255 \\ 1613 & 1134 & 545 & 244 & 94 & 36 \\ 430 & 296 & 131 & 51 & 14 & 1\end{array}$

Figure 2A In the Kaplain-Meier analysis with log-rank test, it showed a better long-term survival rate in the study cohort (Figure 2A).

Figure 2B In the stratification analysis, according to the cumulative Defined Daily Doses (cDDDs), the Kaplain-Meier analysis with log-rank test showed an better long-term survival rate in the users of higher statin cumulative dose. 
Table 2 showed the stratification analyses by age, gender, income, urbanization level, CCI score, inotropic agent use, ICU admission, receiving mechanical ventilation (the latter three procedures were conducted during the index hospitalization). All the subgroups showed that statins use was associated with decreased adjusted HR of mortality. Furthermore, the statins use had a more decreased adjusted HR in the groups of CCI score $\geq 4$, inotropic agents use, ICU admission, and mechanical ventilation which were the weaker populations. 
Table 2 Stratification analyses by age, gender, income, urbanization level, CCI score, inotropic agent use, ICU admission, and mechanical ventilation use.

\section{Statin use}

\begin{tabular}{|c|c|c|c|c|c|c|c|c|}
\hline \multirow{3}{*}{ Variables } & \multicolumn{6}{|c|}{ Statin use } & \multicolumn{2}{|c|}{ Compared with nonusers of statins or $<30$ cDDDD users } \\
\hline & \multicolumn{3}{|c|}{$\begin{array}{c}\text { Nonuser of statins } \\
\text { or }<30 \text { cDDD } \\
(n=36780)\end{array}$} & \multicolumn{3}{|c|}{ Statins user $\geq \mathbf{3 0}$ cDDD } & Crude HR & \multirow[t]{2}{*}{ Adjusted HR } \\
\hline & Event & Person days & $\mathbf{I R}^{\dagger}$ & Event & Person days & $\mathbf{I R}^{\dagger}$ & $(95 \% \mathrm{CI})$ & \\
\hline Total & 12884 & 43841716 & 0.29 & 981 & 10296368 & 0.1 & $0.32(0.3-0.34)^{* * *}$ & $0.29(0.27-0.31)^{* * *}$ \\
\hline \multicolumn{9}{|l|}{ Sex } \\
\hline Female & 6214 & 24657585 & 0.25 & 448 & 5607037 & 0.08 & $0.31(0.28-0.34)^{* * *}$ & $0.3(0.27-0.33) * * *$ \\
\hline Male & 6670 & 19184131 & 0.35 & 533 & 4689331 & 0.11 & $0.32(0.29-0.35)^{* * *}$ & $0.28(0.26-0.31)^{* * *}$ \\
\hline \multicolumn{9}{|l|}{ Age group, years } \\
\hline $18-40$ years & 297 & 4321393 & 0.07 & 17 & 358829 & 0.05 & $0.62(0.38-1.01)$ & $0.49(0.3-0.81)^{* *}$ \\
\hline $40-60$ years & 2186 & 15041306 & 0.15 & 146 & 3167089 & 0.05 & $0.29(0.25-0.35)^{* * *}$ & $0.29(0.24-0.34)^{* * *}$ \\
\hline $60-80$ years & 6309 & 19400649 & 0.33 & 548 & 5529448 & 0.10 & $0.31(0.28-0.33)^{* * *}$ & $0.28(0.26-0.31)^{* * *}$ \\
\hline$>80$ years & 4092 & 5078368 & 0.81 & 270 & 1241002 & 0.22 & $0.3(0.27-0.34)^{* * *}$ & $0.29(0.26-0.33)^{* * *}$ \\
\hline \multicolumn{9}{|c|}{ Insurance premium (NT dollars) } \\
\hline$<20000$ & 8170 & 19745100 & 0.41 & 595 & 5221662 & 0.11 & $0.28(0.26-0.31)^{* * *}$ & $0.27(0.25-0.3)^{* * *}$ \\
\hline $20000-40000$ & 3948 & 18696366 & 0.21 & 339 & 4175637 & 0.08 & $0.36(0.32-0.4)^{* * *}$ & $0.33(0.29-0.36)^{* * *}$ \\
\hline $40000-60000$ & 547 & 3985309 & 0.14 & 33 & 699580 & 0.05 & $0.29(0.2-0.41)^{* * *}$ & $0.26(0.18-0.37)^{* * *}$ \\
\hline$>60000$ & 219 & 1414941 & 0.15 & 14 & 199489 & 0.07 & $0.39(0.23-0.67)^{* * *}$ & $0.35(0.2-0.6)^{* * *}$ \\
\hline \multicolumn{9}{|l|}{ Urbanization level } \\
\hline 1 (highest) & 3243 & 11488076 & 0.28 & 266 & 2778328 & 0.10 & $0.33(0.29-0.37)^{* * *}$ & $0.3(0.26-0.34)^{* * *}$ \\
\hline 2 & 3639 & 13084938 & 0.28 & 255 & 2955044 & 0.09 & $0.3(0.26-0.34)^{* * *}$ & $0.27(0.24-0.3)^{* * *}$ \\
\hline 3 & 1960 & 6728682 & 0.29 & 149 & 1624333 & 0.09 & $0.31(0.26-0.37)^{* * *}$ & $0.28(0.24-0.33)^{* * *}$ \\
\hline
\end{tabular}




\begin{tabular}{|c|c|c|c|c|c|c|c|c|}
\hline 4 & 2084 & 6514345 & 0.32 & 142 & 1563331 & 0.09 & $0.28(0.24-0.34)^{* * *}$ & $0.26(0.22-0.31)^{* * *}$ \\
\hline 5 (lowest) & 1958 & 6025675 & 0.32 & 169 & 1375332 & 0.12 & $0.37(0.31-0.43)^{* * *}$ & $0.35(0.3-0.41)^{* * *}$ \\
\hline \multicolumn{9}{|l|}{ CCI score } \\
\hline 0 & 135 & 2780099 & 0.05 & 11 & 207041 & 0.05 & $1.04(0.56-1.93)$ & $0.82(0.44-1.53)$ \\
\hline 1 & 321 & 4924805 & 0.07 & 32 & 898760 & 0.04 & $0.55(0.38-0.79)^{* *}$ & $0.49(0.34-0.71)^{* * *}$ \\
\hline 2 & 465 & 4036526 & 0.12 & 57 & 957257 & 0.06 & $0.51(0.38-0.67)^{* * *}$ & $0.46(0.35-0.61)^{* * *}$ \\
\hline 3 & 528 & 3404285 & 0.16 & 57 & 914056 & 0.06 & $0.39(0.3-0.52)^{* * *}$ & $0.4(0.3-0.52)^{* * *}$ \\
\hline$\geq 4$ & 11435 & 28696001 & 0.40 & 824 & 7319254 & 0.11 & $0.29(0.27-0.31)^{* * *}$ & $0.27(0.25-0.29)^{* * *}$ \\
\hline \multicolumn{9}{|c|}{ Inotropic agent use } \\
\hline \multicolumn{9}{|c|}{ (During hospitalization) } \\
\hline No & 7290 & 35209544 & 0.21 & 762 & 8366867 & 0.09 & $0.42(0.39-0.45)^{* * *}$ & $0.36(0.34-0.39)^{* * *}$ \\
\hline Yes & 5594 & 8632172 & 0.65 & 219 & 1929501 & 0.11 & $0.2(0.17-0.23)^{* * *}$ & $0.2(0.17-0.22)^{* * *}$ \\
\hline \multicolumn{9}{|c|}{ ICU admission } \\
\hline \multicolumn{9}{|c|}{ (During hospitalization) } \\
\hline No & 6091 & 30259530 & 0.20 & 607 & 7151932 & 0.08 & $0.39(0.36-0.43)^{* * *}$ & $0.34(0.31-0.37)^{* * *}$ \\
\hline Yes & 6793 & 13582186 & 0.50 & 374 & 3144436 & 0.12 & $0.26(0.23-0.29)^{* * *}$ & $0.26(0.23-0.28)^{* * *}$ \\
\hline \multicolumn{9}{|c|}{ Mechanical ventilation } \\
\hline \multicolumn{9}{|c|}{ (During hospitalization) } \\
\hline No & 9021 & 39432964 & 0.23 & 838 & 9490717 & 0.09 & $0.37(0.34-0.39)^{* * *}$ & $0.32(0.3-0.35)^{* * *}$ \\
\hline Yes & 3863 & 4408752 & 0.88 & 143 & 805651 & 0.18 & $0.24(0.2-0.28)^{* * *}$ & $0.25(0.21-0.3)^{* * *}$ \\
\hline \multicolumn{9}{|c|}{ Abbreviations: IR: incidence rates; HR: hazard ratio; CI: confidence interval. } \\
\hline
\end{tabular}


In the sensitivity analysis, we further exclude the weakest patients who expired soon after discharge. That is, we excluded the patients who died within three and six months post-discharge from the index hospitalization of sepsis, both the adjusted HRs remained significant (adjusted HR = 0.35, 95\%CI 0.32-0.37 and adjusted $\mathrm{HR}=0.42$, 95\%CI 0.39-0.45, respectively) for mortality (Supplement Table 1).

\section{Discussion}

In this current study, we demonstrated that the statins use improved the long-term outcome of survived sepsis hospitalization patients. And we further found that statins use may benefit more in the weaker populations, that is, the patients with multiple comorbidities (CCI score $\geq 4$ in this current study) and of higher severity during the sepsis hospitalization (inotropic agents use, ICU admission, and receiving mechanical ventilation). This nation-scale cohort study skipped the previous nonresolved controversy, "effect of statins prior to or during sepsis courses and the associated hospital outcomes", and provided a new perspective in post-sepsis care program.

\section{Major cardiovascular events}

In the case-crossover study by Bohme et al., it demonstrated that the risk of stroke and intracranial hemorrhage remarkably increased post sepsis hospitalization, and the risk increased as the time window got closer to the sepsis event; this relation persisted up to a 1-year period.[15] Similar result was also demonstrated by Ou et al. which further included myocardial infarction, heart failure, and sudden cardiac death as the study endpoints. ${ }^{6}$ A longitudinal cohort study with a followup period of over than 6 years conducted by Wang et al. demonstrated that sepsis patients were at increased risk of mortality post sepsis events and about $70 \%$ of the death post sepsis was caused by cardiovascular or pulmonary diseases.[16] The above studies supports our original hypothesis that among these survived sepsis patients, they were at markedly increased risk of all kinds of cardiovascular diseases with subsequent mortality; statin use may reduce these risk factors and therefore increase the long-term survival.

In the study by Bohme et al., it demonstrated that the post-sepsis younger patients were at a higher risk of ischemic stroke compared with the older patients. Our study also showed a better protective effect of statins in the older populations (>40 years) (adjusted HR $=0.29$ (95\% CI 0.25-0.33)) than in the young population (18-40 years) (adjusted HR $=0.49$ (95\% CI 0.3-0.81)). This may be explained by the multi-etiology of young strokes post sepsis rather than baseline comorbidity burdens, such as atherosclerosis, associated with increased age.

\section{Anti-microbial effects}

Recurrence and new-onset of infection conditions were a crucial issue in post-sepsis survivors. It still remains a controversy that if statin therapy was associated with a better outcome during sepsis hospitalization. In the meta-analysis by Wan et al, they found that in the randomized controlled trials, statin use did not significantly decrease the hospital mortality during a sepsis hospitalization; however, the observational studies demonstrated that statin use were associated with a significant decrease in hospital mortality.[12] In the above-mentioned clinical trials, statins of interest were usually de novo prescribed to the study populations during the sepsis hospitalization and the placebo users were regarded as the comparison cohorts. However, in observational studies, the statin users were usually pretreated statin users and were further compared with the non-statin users. In a national cohort study by Caffrey et al., it demonstrated in detail that among the patients with Staphylococcus aureus bacteremia, the continuation of statin therapy among pretreated statin users was associated with significant beneficial effects on 30-day hospital mortality, but not in de novo statin users or in pretreated statin users who did not continue statin therapy after admission. [ $\left.{ }^{17}\right]$ From these studies, it was reasonable to infer that statin therapy should continue for a period up to an effective cumulative dose to exert its protective effects in sepsis. And this is relatively consistent with our study that the protective effect of statins was positively proportional to the cDDDs. 
Prior studies had demonstrated the effect of statins to prevent infections and to reduce sepsis severity via the immunomodulatory and anti-inflammatory effects. Masadeh et al. demonstrated the antibacterial activity of statins between the atorvastatin, simvastatin, and rosuvastatin. $\left.{ }^{18}\right]$ McDowell et al. reported that simvastatin was protective during Staphylococcus aureus pneumonia.[19] In the study by Su et al., it reported that statin use was associated with a lower risk of Mycobacterium tuberculosis infection. $\left[{ }^{13}\right]$ Liappis et al. demonstrated the protective effect of statins on mortality in patients with bacteremia, and not only in Staphylococcus aureus but also in aerobic gram-negative bacilli bacteremia. $\left.{ }^{20}\right]$ The basic laboratory studies also showed similar results to the clinical studies about the antimicrobial effects of statins. Statt et al. reported that statins could enhance the cellular resistance against bacterial pore-forming toxins in airway epithelial cells. $\left.{ }^{21}\right]$ In the study by Graziano et al., it also showed that simvastatin could be as a potential drug against Staphylococcus aureus biofilm.[22] These studies may support our second hypothesis that statin use improved the long-term outcome post sepsis by its potential antibacterial effect and therefore reduced the recurrent infections.

\section{Healthy user bias}

"Healthy user bias" was frequently proposed as an important bias in the retrospective study of "statins in sepsis outcomes". It was described as the higher health awareness and healthier lifestyle in the patients of statin-users compared with non-statin users. According to this theory, statin users were more likely to seek out preventive health services, such as screening tests and vaccinations. ${ }^{23}$ However, it was quite difficult to measure the lifestyle factors, disease prevention behaviors and drugs compliance in observational studies. To reduce the impact of confounding from the "healthy user bias", we used the individual insurance premium as a proxy to adjust for socioeconomic status. And the propensity score matching which included the baseline comorbidities, income, and urbanization level as variables to further reduce the selection bias related to the "healthy user bias". This statistical methodology also helps the observation studies simulate the randomized control trials.

Indication bias of statins

The indication bias of statin may be also challenged in this study. In Taiwan, statins were not available over-the counter; the physicians' decision of statin treatment should not only follow the treatment guideline of the specific diseases but also the payment regulations by the NHIA (Supplement Table 2). If the prescriptions were against the rules, the NHIA could not only refuse to pay the medical fee but also punished the physicians with a maximal 100-fold rebound (because the national health insurance program was a single-payer, compulsive insurance coverage policy in Taiwan, and the NHIA had the full authority to control all the medical facilities and healthcare professionals).

Strengths

Our study had strengths. First, this nation-scale study provided a large sample size and longer observation time as mentioned in the title "long-term" rather than the short interval of one year or even shorter period from admission to discharge. Second, the included patients were categorized into four groups according to the cDDDs of statins during the follow-up period (non-users or users $<30$ cDDDs, users of 30-180, 180-365, and >365 cDDDs). This categorization helped examine the possible dose-response effect in this current observational study. Third, we conducted the sensitivity analyses, which excluded the survived sepsis hospitalization patients who died within the three and six months post sepsis. Most survived sepsis patients died not far from discharge because of multiple factors. From the sensitivity analyses, we could further examine the long-term protective effect of statins.

\section{Limitations}


The major limitations of this study comes from the unavailable data of enrolled sepsis patients, that is, the serial lipid profiles, and body mass index which may change over time. However, in the real world, these data are also hardly to collect once the follow-up time is longer. Second, this work shares the universal limitations of all studies from databases that drug dispensing or prescribing are not the actual drug intake. Third, we did not have the final information of mortality causes of the enrolled patients, such as major adverse cardiovascular events or recurrent infections. We could only explain the conclusions by referring to previous studies.

\section{Conclusions}

Statins use may improve the long-term outcomes of survived sepsis patients via complex mechanisms, especially in the weaker populations, such as of multiple comorbidities and higher severity during sepsis hospitalization. Further randomized control trial with long-term follow up should be conducted to further confirm our conclusions.

\section{Declarations}

Abbreviations:

CCI score: Charlson comorbidity index score; cDDDs: cumulative Defined Daily Doses; CIs: confidence intervals; HRs: hazard ratios; ICD-9-CM: International Classification of Diseases, Ninth Revision, Clinical Modification; ICU: intensive care unit; MACEs: major cardiovascular events; NHIA: National Health Insurance Administration; NHIRD: National Health Insurance Research Database; NT: New Taiwan;

Ethics Approval and Consent to Participate:

This study was conducted by using the NHIRD in Taiwan. The NHIRD contains deidentified secondary data for research; our study was exempted from the requirement of informed consent from participants. This study was approved by the Institutional Review Board of China Medical University (CMUH104-REC2-115).

Consent for publication:

Not applicable.

Availability of data and material:

Please contact the author for data requests.

Acknowledgements:

We thank the Biostatistics Task Force of Taichung Veterans General Hospital, Taichung, Taiwan, Republic of China, for their assistance and advice in the statistical analyses.

\section{Author Contributions:}

The authors' individual contributions are as follows. Conception and design: M-SH, S-HL, Y-TL, and C-KH. Data analysis and interpretation: Vivian C-RH, J-HC, and C-JS. Manuscript writing: M-SH. Final approval and critical revision: P-CC and S-YH. All the authors read and approved the final manuscript.

Funding/Support: 
This study is supported in part by Taiwan Ministry of Health and Welfare Clinical Trial Center (MOHW107-TDU-B-212-123004), China Medical University Hospital, Academia Sinica Stroke Biosignature Project (BM10701010021), MOST Clinical Trial Consortium for Stroke (MOST 1062321-B-039-005-).", Tseng-Lien Lin Foundation, Taichung, Taiwan, and Katsuzo and Kiyo Aoshima Memorial Funds, Japan.

The funders had no role in the study design, data collection, analysis, decision to publish, or preparation of the manuscript. No additional external funding was received for this study.

\section{Potential Conflicts of Interests (Competing interests):}

None of the authors have any conflicts of interest to disclose. We confirm that we have read the Journal's position on issues involved with unethical publication and affirmed that this study is consistent with those guidelines.

\section{References}

1. Vincent, J. L.; Marshall, J. C.; Namendys-Silva, S. A.; Francois, B.; Martin-Loeches, I.; Lipman, J.; Reinhart, K.; Antonelli, M.; Pickkers, P.; Njimi, H.; Jimenez, E.; Sakr, Y., Assessment of the worldwide burden of critical illness: the intensive care over nations (ICON) audit. Lancet Respir Med 2014, 2 (5), 380-6.

2. Fleischmann, C.; Scherag, A.; Adhikari, N. K.; Hartog, C. S.; Tsaganos, T.; Schlattmann, P.; Angus, D. C.; Reinhart, K., Assessment of Global Incidence and Mortality of Hospital-treated Sepsis. Current Estimates and Limitations. Am J Respir Crit Care Med 2016, 193 (3), 259-72.

3. Singer, M.; Deutschman, C. S.; Seymour, C. W.; Shankar-Hari, M.; Annane, D.; Bauer, M.; Bellomo, R.; Bernard, G. R.; Chiche, J. D.; Coopersmith, C. M.; Hotchkiss, R. S.; Levy, M. M.; Marshall, J. C.; Martin, G. S.; Opal, S. M.; Rubenfeld, G. D.; van der Poll, T.; Vincent, J. L.; Angus, D. C., The Third International Consensus Definitions for Sepsis and Septic Shock (Sepsis-3). Jama 2016, 315 (8), 801-10.

4. Prescott, H. C.; Langa, K. M.; Liu, V.; Escobar, G. J.; Iwashyna, T. J., Increased 1-year healthcare use in survivors of severe sepsis. Am J Respir Crit Care Med 2014, 190 (1), 62-9.

5. Shankar-Hari, M.; Ambler, M.; Mahalingasivam, V.; Jones, A.; Rowan, K.; Rubenfeld, G. D., Evidence for a causal link between sepsis and long-term mortality: a systematic review of epidemiologic studies. Crit Care 2016, 20, 101.

6. Ou, S. M.; Chu, H.; Chao, P. W.; Lee, Y. J.; Kuo, S. C.; Chen, T. J.; Tseng, C. M.; Shih, C. J.; Chen, Y. T., LongTerm Mortality and Major Adverse Cardiovascular Events in Sepsis Survivors. A Nationwide Population-based Study. Am J Respir Crit Care Med 2016, 194 (2), 209-17.

7. Chao, P. W.; Shih, C. J.; Lee, Y. J.; Tseng, C. M.; Kuo, S. C.; Shih, Y. N.; Chou, K. T.; Tarng, D. C.; Li, S. Y.; $\mathrm{Ou}, \mathrm{S}$. M.; Chen, Y. T., Association of postdischarge rehabilitation with mortality in intensive care unit survivors of sepsis. Am J Respir Crit Care Med 2014, 190 (9), 1003-11.

8. Desai, S. V.; Law, T. J.; Needham, D. M., Long-term complications of critical care. Crit Care Med 2011, 39 (2), 371-9.

9. Linder, A.; Guh, D.; Boyd, J. H.; Walley, K. R.; Anis, A. H.; Russell, J. A., Long-term (10-year) mortality of younger previously healthy patients with severe sepsis/septic shock is worse than that of patients with nonseptic critical illness and of the general population. Crit Care Med 2014, 42 (10), 2211-8.

10. Almog, Y., Statins, inflammation, and sepsis: hypothesis. Chest 2003, 124 (2), 740-3.

11. Arnaud, C.; Mach, F., Potential antiinflammatory and immunomodulatory effects of statins in rheumatologic therapy. Arthritis Rheum 2006, 54 (2), 390-2.

12. Wan, Y. D.; Sun, T. W.; Kan, Q. C.; Guan, F. X.; Zhang, S. G., Effect of statin therapy on mortality from infection and sepsis: a meta-analysis of randomized and observational studies. Crit Care 2014, 18 (2), R71.

13. Su, V. Y.; Su, W. J.; Yen, Y. F.; Pan, S. W.; Chuang, P. H.; Feng, J. Y.; Chou, K. T.; Yang, K. Y.; Lee, Y. C.; Chen, T. J., Statin Use Is Associated With a Lower Risk of TB. Chest 2017, 152 (3), 598-606.

14. Lee, C. C.; Lee, M. G.; Hsu, T. C.; Porta, L.; Chang, S. S.; Yo, C. H.; Tsai, K. C.; Lee, M., A Population-Based Cohort Study on the Drug-Specific Effect of Statins on Sepsis Outcome. Chest 2018, 153 (4), 805-815.

15. Boehme, A. K.; Ranawat, P.; Luna, J.; Kamel, H.; Elkind, M. S., Risk of Acute Stroke After Hospitalization for Sepsis: A Case-Crossover Study. Stroke 2017, 48 (3), 574-580. 
16. Wang, H. E.; Szychowski, J. M.; Griffin, R.; Safford, M. M.; Shapiro, N. I.; Howard, G., Long-term mortality after community-acquired sepsis: a longitudinal population-based cohort study. BMJ Open 2014, 4 (1), e004283. 17. Caffrey, A. R.; Timbrook, T. T.; Noh, E.; Sakoulas, G.; Opal, S. M.; Nizet, V.; LaPlante, K. L., Evidence To Support Continuation of Statin Therapy in Patients with Staphylococcus aureus Bacteremia. Antimicrob Agents Chemother 2017, 61 (3).

18. Masadeh, M.; Mhaidat, N.; Alzoubi, K.; Al-Azzam, S.; Alnasser, Z., Antibacterial activity of statins: a comparative study of atorvastatin, simvastatin, and rosuvastatin. Ann Clin Microbiol Antimicrob 2012, 11, 13.

19. McDowell, S. A.; Ma, Y.; Kusano, R.; Akinbi, H. T., Simvastatin is protective during Staphylococcus aureus pneumonia. Curr Pharm Biotechnol 2011, 12 (9), 1455-62.

20. Liappis, A. P.; Kan, V. L.; Rochester, C. G.; Simon, G. L., The effect of statins on mortality in patients with bacteremia. Clin Infect Dis 2001, 33 (8), 1352-7.

21. Statt, S.; Ruan, J. W.; Hung, L. Y.; Chang, C. Y.; Huang, C. T.; Lim, J. H.; Li, J. D.; Wu, R.; Kao, C. Y., Statinconferred enhanced cellular resistance against bacterial pore-forming toxins in airway epithelial cells. Am J Respir Cell Mol Biol 2015, 53 (5), 689-702.

22. Graziano, T. S.; Cuzzullin, M. C.; Franco, G. C.; Schwartz-Filho, H. O.; de Andrade, E. D.; Groppo, F. C.; Cogo-Muller, K., Statins and Antimicrobial Effects: Simvastatin as a Potential Drug against Staphylococcus aureus Biofilm. PLoS One 2015, 10 (5), e0128098.

23. Brookhart, M. A.; Patrick, A. R.; Dormuth, C.; Avorn, J.; Shrank, W.; Cadarette, S. M.; Solomon, D. H., Adherence to lipid-lowering therapy and the use of preventive health services: an investigation of the healthy user effect. Am J Epidemiol 2007, 166 (3), 348-54. 